\title{
An Estimate of Fetal Autonomic State by Spectral Analysis of Fetal Heart Rate Fluctuations
}

\author{
J. KARIN, M. HIRSCH, AND S. AKSELROD
}

The Abramson Institute of Medical Physics, Sackler Faculty of Exact Sciences, Tel Aviv University [J.K.. S.A.]: and Department of Obstetrics and Gynecology. Beilinson Medical Center, Petah Tikva, and the Dan District of Kupat-Holim Haklalit, Israel [M.H.]

\begin{abstract}
The assessment of the functional state of the autonomic nervous system (ANS) in real time, by means of spectral analysis of fetal heart rate variability, may serve to improve the diagnosis of pathologic conditions of importance to the perinatologist. The combination of two approaches, namely an efficient method for detecting fetal ECG from the abdominal maternal signal, followed by spectral analysis of heart rate variability, is tested as a new noninvasive tool to assess fetal viability in real time. This study demonstrates a pattern of ANS development via the spectral contents of heart rate variability. It is shown that during "quiet state," the "young" fetuses (gestational age $=23.5 \pm 1$ wk) present twice as much power of heart rate fluctuations at all frequencies from 0.2 to 1.0 $\mathrm{Hz}$ as "mature" fetuses (gestational age $=39.75 \pm 1.5$ wk). This finding is coherent with the evolution of a stable and mature ANS activity. At frequencies below $0.1 \mathrm{~Hz}$, a $1 / \mathrm{f}^{\alpha}$ power law relationship $\left(\alpha=0.85, r^{2}>0.9\right)$ between spectral density and frequency is displayed in the two age groups. A respiratory peak has been observed in some of the short (64-s) traces we analyzed. However, no respiratory peak was ever observed in a long (256-s) trace, due to the episodic nature of the fetal breathing and immaturity of the ANS. (Pediatr Res 34: 134-138, 1993)
\end{abstract}

\section{Abbreviations}

AECG, abdominal ECG
HR, heart rate
FHR, fetal heart rate
HRV, heart rate variability
GA, gestational age
ANS, autonomic nervous system
RP, respiratory peak

Thorough understanding of the development of the fetal ANS is still lacking. This may be mainly attributed to the inaccessibility of the fetus for direct measurements. In recent years, the development of noninvasive means, such as electronic FHR monitoring and real-time ultrasonography, enlarged the basic knowledge concerning fetal heart functions as well as body movements, respiratory activity, and behavioral patterns. Nonetheless, more information is still required to improve our ability for early detection and avoidance of adverse perinatal outcome.

Received February 21, 1992; accepted March 12, 1993.

Correspondence and reprint requests: S. Akselrod, School of Physics and Astronomy, Faculty of Exact Sciences, Tel Aviv University, Ramat Aviv, 69978, Israel.
In adults and newborns, HRV as estimated by spectral analysis is known to reflect the activity of various components of the ANS (1-3), i.e. each of the two autonomic inputs into the sinoatrial node, sympathetic and parasympathetic, contribute to the HR power spectrum in different, well-determined frequency ranges. This frequency specificity enables an estimation of parasympathetic activity as well as the evaluation of the balance between sympathetic and parasympathetic tone. By applying spectral analysis to HRV, autonomic control has been delineated in a cardiovascular system of adults under normal conditions as well as in various pathologic states that involve suspected autonomic malfunction (4-7). In adults, a high-frequency peak (RP) in the HRV power spectrum is associated (8) with respiratory activity.

Fetal breathing movements have been detected as early as 11 wks of gestation. They remain irregular during the first half of the pregnancy and become slower and more regular during the last weeks before delivery (9). They are known to enhance HRV and may usually persist in both "wakefulness" and "sleep" states $(10,11)$. These behavioral patterns occur in the normal pregnancy and reflect the development of the fetal ANS. Therefore, the features of the HRV power spectrum in the fetus might provide us with a pattern of the development and functioning of the fetal autonomic system. Relative changes in respiratory versus low-frequency fluctuations may reveal a gradual process of ANS maturation or a state of disease.

A novel algorithm for on-line detection of FHR (12) permits an accurate beat-to-beat detection of the FHR from an ECG signal obtained on the maternal abdominal wall. This, combined with spectral analysis of $\mathrm{HRV}$, is presented as a noninvasive means to assess fetal ANS activity and dynamic control in real time. This approach might allow the investigation of "behavioral" states of the fetus as well as detection of "breathing" activity. It may provide us with a potential tool for early detection of pathologic processes within the ANS, such as fetal distress, postmaturity syndrome, and intrauterine growth retardation, known to be associated with reduced fetal body and breathing movements (9) and changes in FHR patterns (13). For example the likelihood of uterine contractions to progress and to end with premature delivery is associated with the presence of depressed fetal breathing (14). Such problems, as well as the investigation of fetal ANS development, may be better approached by evaluating the spectral contents of the fetal HRV, noninvasively obtained from an AECG.

A classic problem in physics is the $1 / f^{\prime r}$ relation between frequency and power density found in the low-frequency range of the power spectrum of many systems in general and in physiologic systems $(15,16)$ such as EEG $\alpha$-wave, heart beat period, and density modulation of action potentials in the nerve axon. The origin of this widespread phenomenon is probably connected with spatial degrees of freedom, leading the system 
into barely stable states and to the inverse power law for temporal fluctuations (17). Compliance of fetal HRV with the known 1/ $f^{\prime \prime}$ relationship will support the validity of the FHR detection process, and the $\alpha$-parameter value may add another parameter for the description of the fetal nervous system.

\section{MATERIALS AND METHODS}

Outline of algorithm for fetal ECG detection. The approach we use for the FHR detection algorithm is based on the cancellation of the maternal contribution in both the first and second derivatives of the AECG, in contrast to other cancellation procedures generally performed on the AECG signal itself.

The fetal QRS detection method we designed (12) and applied here includes the steps outlined in Figure 1. Accurate detection of fetal QRS complexes is achieved, and a series of fetal R-R intervals can be created, as well as an AECG signal where each fetal beat is indicated.

Patient recruitment and data collection. Subjects were selected at random from a healthy population undergoing routine pregnancy follow-up. 1) Details about maternal age and GA (completed weeks starting from the 1 st $\mathrm{d}$ of the last known menstrual period) were noted. 2) Patients presenting maternal or fetal complications or taking medications, other than iron or vitamins, were excluded from the study. 3) Each recording session took place between 0900 and $1200 \mathrm{~h}$, with the patient, after at least $10 \mathrm{~min}$ rest, in supine position.

Each subject was subjected to a 5-min AECG recording (combined fetal-maternal signal) measured from the maternal abdomen, on a TEAC R-71 data cassette recorder (TEAC Corp. of America, CA), to which the output of a sensitive low-noise ECG amplifier (qualified for human signal monitoring) was connected. Only one ECG lead was recorded, implemented with three electrodes.

Data analysis. The analysis requires the AECG signal to be fed into an analog-to-digital converter (A/D Labmaster PGL-40, Scientific Solutions, Inc., OH) of a personal computer (IBM-AT or compatible). The AECG signal was sampled at a rate of 300 $\mathrm{Hz}$, and the algorithm for FHR detection from the maternalfetal signal was then applied.

Because the fetal heart beat detection is not always perfect. and some fetal $R$ waves may not be detected, the series of $R-R$ intervals goes through an automatic correction procedure that detects abnormal values of FHR or abrupt FHR variations and correcting abnormal $\mathrm{R}-\mathrm{R}$ intervals, while conserving the absolute time. The R-R series is then submitted to an algorithm (18) that creates equally time-spaced FHR values. It simultaneously performs digital smoothing and low-pass filtering (at $2 \mathrm{~Hz}$ ), using a moving average window.

This procedure results in a smooth time series of FHR values, (Fig. 2) computed at equal time intervals of $0.25 \mathrm{~s}(4 \mathrm{~Hz})$. The

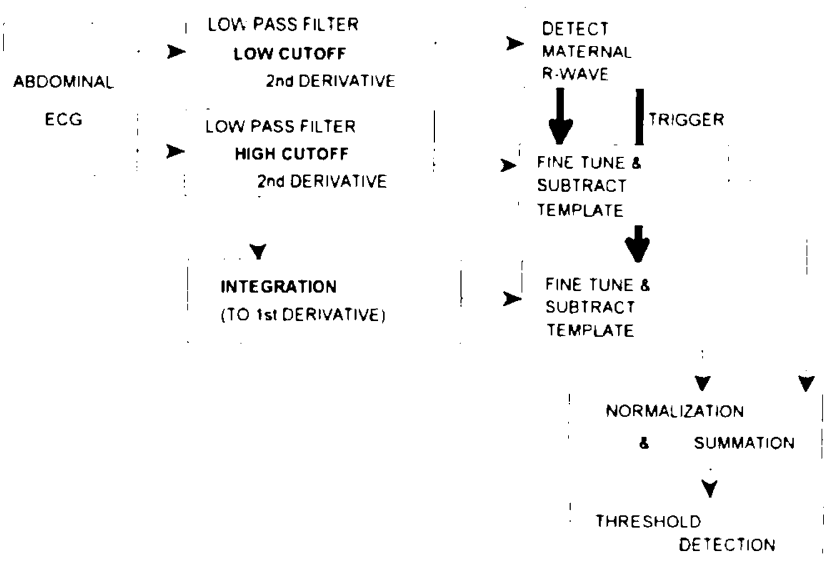

Fig. 1. Block diagram of fetal $\mathrm{R}$ wave detection process, using second derivative estimation and cancellation of the maternal contribution.

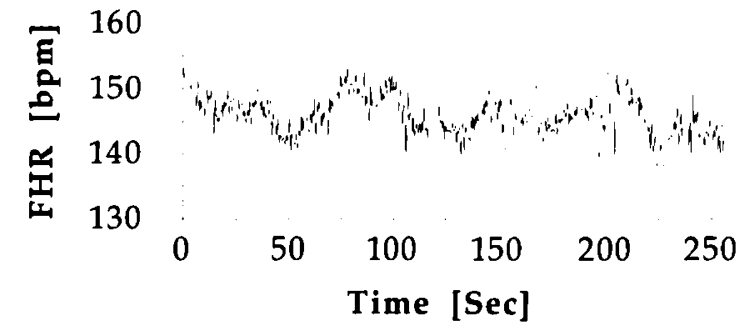

Fig. 2. Fetal HR from a pregnancy of 38 gestational wk.

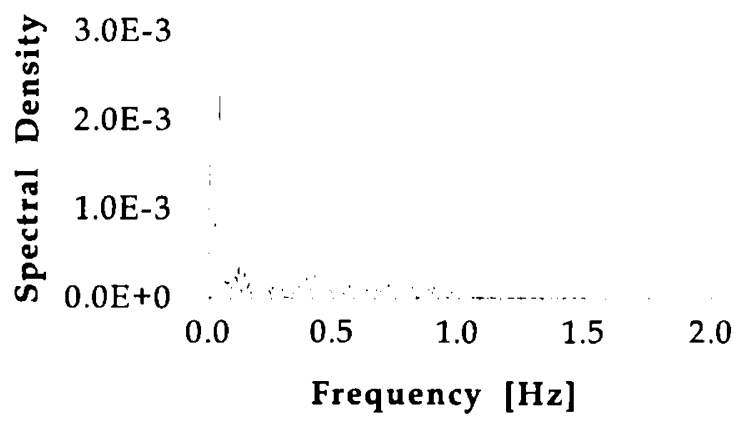

Fig. 3. Power spectrum of fetal HR variations, taken from fetal HR in Figure 2 (256-s trace).

\section{$2.0 \mathrm{E}-4$}

\section{$1.0 \mathrm{E}-4$}

$0.0 \mathrm{E}+0$

\section{$\stackrel{2}{\infty} 2.0 \mathrm{E}-4$

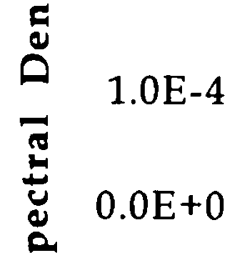 \\ on}

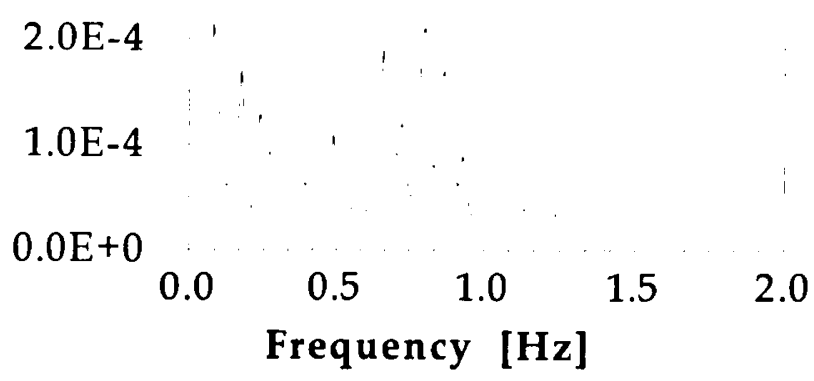

Fig. 4. Power spectra of fetal HR variations, taken from 64-s subtraces of the fetal HR in Figure 2. A high-frequency peak may be observed at the $0.4-$ to $1.0-\mathrm{Hz}$ band.

frequency range we are interested in, as well as the possible modulation mechanisms (the fastest being breathing movements) acting on FHR, do not exceed $2 \mathrm{~Hz}$. Therefore, a sampling rate of $4 \mathrm{~Hz}$ is sufficient for our purpose. Next, this fluctuating FHR time series undergoes a fast Fourier transform to allow the computation of the power spectrum (19) up to a frequency of 2 Hz (Fig. 3). 


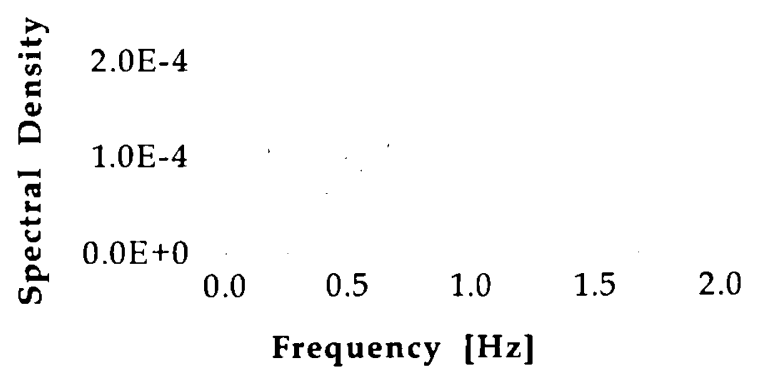

Fig. 5. Average HRV power spectrum of the three 64-s subtraces presented in Figure 4.

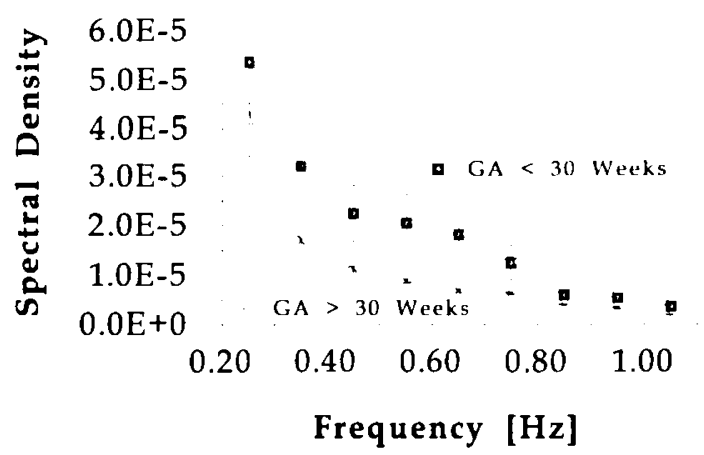

Fig. 6. Average integrals $(0.1-\mathrm{Hz}$ wide) over $\mathrm{HRV}$ power spectra of 64-s subtraces taken during sleep state (FHR variance $<15 \mathrm{bpm}^{2}$ ) from young pregnancies $(\mathrm{GA}=23.5 \pm 1 \mathrm{wk}, n=10)$ and mature pregnancies $(\mathrm{GA}=39.75 \pm 1.5 \mathrm{wk}, n=22)$.

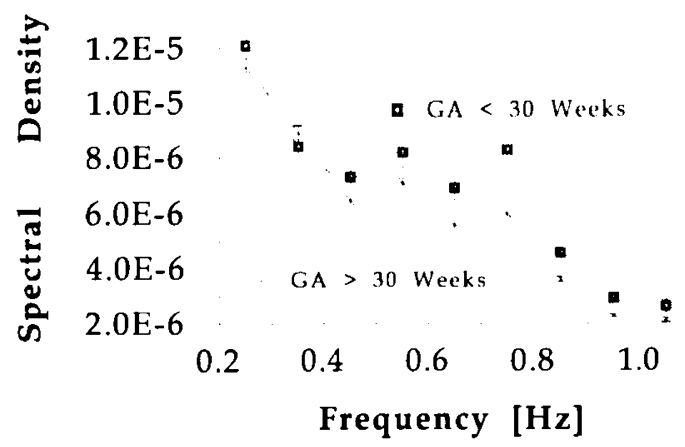

Fig. 7. Average integrals $(0.1-\mathrm{Hz}$ wide $)$ over $\mathrm{HRV}$ power spectra of 64-s subtraces taken during active state (FHR variance $>30 \mathrm{bpm}^{2}$ ) from young pregnancies $(\mathrm{GA}=24 \pm 0.8 \mathrm{wk}, n=3)$ and mature pregnancies $(\mathrm{GA}=39.75 \pm 1.5 \mathrm{wk}, n=16)$.

Integrals over predefined frequency bands of the power spectrum are the quantitative parameters we use to evaluate the intensity of FHR fluctuations. The value of these integrals is the main quantitative information that we use to assess the autonomic activity in the fetus. The power of HRV in the relevant frequency bands is integrated, normalized with respect to the width of the integration band, and compared between subjects or within subjects at different behavioral states. The frequency bands we chose in this study are simply adjacent bands, of 0.1 $\mathrm{Hz}$ width each, covering the range from 0.2 to $1.1 \mathrm{~Hz}$.

Determining the optimal trace length for analysis. The power spectrum of an unsteady signal tends to diffuse over wide frequency bands and is therefore difficult to characterize. Because breathing movements are known to appear episodically and usually for periods of 30 to $60 \mathrm{~s}(20)$, the power spectrum was analyzed from 64-s subtraces of the original trace (Fig. 4).

Although a power spectrum from a shorter subtrace is less informative in the low-frequency range, it allows us to investigate traces with higher likelihood of homogenous fetal activity, and thus provides more reliable high-frequency information. For short traces of FHR with clear indication of high-frequency content (Fig. 4), we can compute an average power spectrum of subtraces obtained from the same fetus, aiming at the enhancement of specific consistent features of the spectrum (Fig. 5).

For example, each of the HRV power spectra of three successive 64-s subtraces shown in Figure 4 display a peak around 0.7 $\mathrm{Hz}$, however with varying amplitude and width. The average power spectrum in Figure 5 presents a more defined highfrequency peak than the HRV power spectrum of the 256-s trace (Fig. 3).

Statistical analysis. Cyclic variations in fetal states are of importance to the study. The level of activity ("sleep state") of the fetus is represented by the standard deviation of FHR. The changing level of activity clearly influences the power spectra of FHR. Unless taken into consideration, this activity modulation may result in large within-subject variation.

To resolve this problem, we conducted a comprehensive statistical analysis to take all the available parameters such as integration frequency band, fetal sleep state, and age into consideration. We used the unbalanced analysis of variance and covariance with repeated measures to determine the influence of each of the above three variables on the spectral integrals and the interactions among these variables. Due to variations in fetal activity state and ECG signal quality, each fetus has a different number of valid subtraces and thus of measurements. The analysis takes into account any given number of measurements and computes the relative contribution for each fetus accordingly.

The statistical analysis generated a system of nine linear equations, predicting the values of the power spectrum integrals as a function of the fetal GA, integration frequency band, and FHR $\mathrm{SD}$. The fit of the model defined by these linear equations was tested by the likelihood test. These tests allow us to compare results obtained from the different age groups of fetuses and to determine the significance of the impact of the age factor on the spectral integrals.

The statistical analysis is a multistep, complicated process that salculates simultaneously the various interactions among the factors affecting the power spectrum of fetal HRV. It demonstrates the difference between the ANS activity of "young" and "mature" fetuses, taking into account all various levels of fetal activity. Therefore, independently of this statistical analysis, to visualize the effect of GA, we show the average spectral integrals for young and mature fetuses. The different sleep and active states were separated into graphs for low activity (FHR variance $<15 \mathrm{bpm}^{2}$ ) and high activity (FHR variance $>30 \mathrm{bpm}^{2}$ ) graphs. For these graphs, only one subtrace per activity state was taken from each subject. When multiple subtraces were available, the subtraces with the lowest and the highest FHR variance were selected.

\section{RESULTS}

Recordings from 10 young pregnancies $(\mathrm{GA}=23.5 \pm 1 \mathrm{wk})$ and 22 mature pregnancies $(\mathrm{GA}=39.75 \pm 1.5 \mathrm{wk})$ were analyzed. Figures 2 and 3 present a typical trace of FHR and its HRV power spectrum. The spectrum is calculated from the entire period of $256 \mathrm{~s}$ to achieve a good resolution in the low-frequency range. In Figures 4 and 5, the advantage of shorter subtraces is demonstrated. The power spectrum of selected short subtraces reveals a high-frequency peak, whereas a full-length trace does not. The selected subtraces may have a common activity state, and therefore a similar power spectra.

To demonstrate the changes in ANS activity throughout pregnancy and to determine the influence of fetal age and sleep state (or level of fetal activity), an unbalanced analysis of variance and covariance with repeated measures was performed three times: once for the combined group of all fetuses to establish the overall trend, and then separately for the two age groups (young and mature fetuses) to determine the deviation of each group from 
that overall trend. Each time the fit of the model (the set of linear equations predicting the values of the spectral integrals) to the measured data was tested by means of likelihood test. and the results were interpreted via a $\chi^{2}$ test. This test resulted in a significant difference $(p<0.00006)$ between the young and the mature fetuses, thus indicating that fetal age significantly affects the pattern of HRV power spectrum.

Figures 6 and 7 display the average integrals of the HRV power spectrum for two groups of fetuses: young and mature pregnancies, during low- and high-activity periods. Sixty-four s long subtraces of FHR were used to calculate the high- $(\geq 0.2 \mathrm{~Hz})$ frequency part of the spectra and seven integrals, each $0.1 \mathrm{~Hz}$ wide, cover the range up to $1.1 \mathrm{~Hz}$. Figure 6 displays the average integrals for the young $(\mathrm{GA}=23.5 \pm 1 \mathrm{wk}, n=10)$ and mature pregnancies $(\mathrm{GA}=39.75 \pm 1.5 \mathrm{wk}, n=22)$ during low-activity sleep state periods (FHR variance $<15 \mathrm{bpm}^{2}$ ), and Figure 7 displays the average integrals for the young $(\mathrm{GA}=24 \pm 0.8 \mathrm{wk}$. $n=3)$ and mature pregnancies $(\mathrm{GA}=39.75 \pm 1.5 \mathrm{wk}, n=16)$ during high-activity periods (FHR variance $>30 \mathrm{bpm}^{2}$ ).

Figures 6 and 7 depict the difference between young and mature fetuses. It is visible that young fetuses display more power of $\mathrm{HR}$ variations than mature fetuses, and that both groups obviously present significantly higher power during activity periods (Fig. 7) compared with sleep states (Fig. 6).

The $1 / \mathrm{f}$ relationship between spectral density and frequency was tested. Because HRV has been shown to comply with the power law relationship at frequencies under $0.1 \mathrm{~Hz}$, full traces of $256 \mathrm{~s}$ were used to calculate the low-frequency $(<0.2 \mathrm{~Hz})$ range to provide a good low-frequency resolution. Figure 8 presents a logarithmic scale plot of the average normalized integrals versus the frequency in the low-frequency range. A logarithmic fit for the data in Figure 8 results in a slope of $\alpha=$ $-0.85\left(r^{2}=0.99, p<0.005\right)$ for the mature pregnancies and $\alpha$ $=-0.84\left(r^{2}=0.91, p<0.05\right)$ for the young pregnancies.

\section{DISCUSSION}

Continuous long-term (256-s) traces of FHR (Fig. 2) from AECG recordings can be obtained by using our fetal ECG detection (12) and FHR correction algorithms. The corresponding power spectra (Fig. 3) do not reveal a clear peak in the highfrequency range, which may be attributed to fetal breathing activity. However, shorter subtraces, the duration of which is of the same value as the breathing episodes of the fetus, have more chances to exhibit a high-frequency peak (Fig. 4). A peak in the HRV power spectrum at the frequency of respiration is observed in adults (8) around $0.3 \mathrm{~Hz}$, and in newborn babies (21-23) in the range of 0.4 to $0.8 \mathrm{~Hz}$. In the fetus, an RP is observed in some of the short 64-s traces (Figs. 4 and 5), although not as powerful and focused as in adults. It is widely dispersed $( \pm 0.3)$

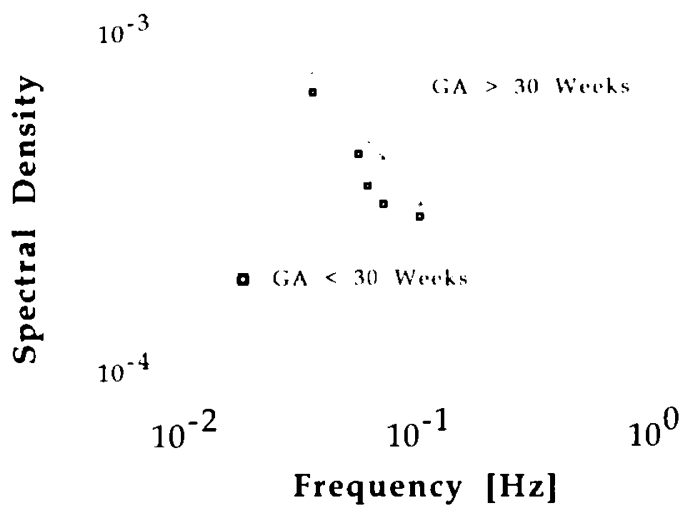

Fig. 8. Log density i's. log frequency of five average HRV power spectrum integrals, taken from 256-s FHR traces of young pregnancies $\left(\mathrm{GA}=23.5 \pm 1 \mathrm{wk}, n=10, r^{2}=0.91, p<0.05\right)$ and mature pregnancies $\left(\mathrm{GA}=39.75 \pm 1.5 \mathrm{wk}, n=22, r^{2}=0.99, p<0.005\right)$. around the $0.7-\mathrm{Hz}$ center. This indicates faster and less organized breathing movements than in adults and conforms with fetal respiratory rate, reported $(20)$ to be $49 \pm 10$ breaths $/ \mathrm{min}$. Average values of integrals over fixed-frequency bands of the HRV power spectrum were calculated and plotted for young and mature fetuses in an active state (FHR variance $>30 \mathrm{bpm}^{2}$ ) in Figure 7 and in sleep state (FHR variance $<15 \mathrm{bpm}^{2}$ ) in Figure 6 . No RP can be observed while fetuses are in an active state (high FHR variability), but during sleep state, two peaks are present at 0.5 to $0.6 \mathrm{~Hz}$ and 0.7 to $0.8 \mathrm{~Hz}$, probably due to bimodal distribution of breathing rates in the traces included in the average.

A similar RP at 0.7 to $0.95 \mathrm{~Hz}$ was reported by Divon et al. (24) in a study performed invasively on human fetuses after spontaneous rupture of membranes. Dykes 't al. (25). Kitney (26), and Giddens and Kitney (27) reported respiratory sinus arrhythmia to be associated with breathing amplitude and RP to be observed in infants but scarcely in very young (1- to 3-d-old) neonates.

Bearing in mind that fetal breathing activity exists only about $25 \%$ (20) of the time and for periods of 30 to $60 \mathrm{~s}$, it is understandable why processing too long a trace may blur an RP if present. This resembles the cancellation of the RP in a 24-h HRV power spectrum versus a 5-min HRV power spectrum in adults (16). Therefore, subtraces of $64 \mathrm{~s}$ were used to produce power spectra, assuming that in such short traces the data have a greater likelihood to present a more stable state of fetal activity. Using this modality of analysis. some of the traces showing no sign of RP in the 256-s spectral analysis unveiled an RP (Figs. 4 and 5) in the shorter subtraces of $64 \mathrm{~s}$. In a study performed at our center, preterm and full-term newborns displayed a dispersed $R P$ in HRV power spectra, even in cases in which they exhibited a clear RP in the direct respiratory power spectrum. Thus, fetal $R P$ is bound to be wide and dispersed. Testing fetal HRV power spectrum density for the $1 / f^{\prime}$ relationship, both groups present essentially the same $\alpha=-0.85$ behavior, comparable with the slope of $\alpha=-1.02$ reported (16) in adults. This substantiates the validity of our fetal $R$ wave detection and FHR correction procedure and provides an additional description of fetal HRV, but presents no new information about the process of fetal ANS maturation.

In an attempt to define a parameter that represents the degree of development of the fetal ANS based on its HRV power spectra. the integrals over constant frequency bands of HRV power spectrum were tested. We used the unbalanced analysis of variance and covariance with repeated measures statistical test. which incorporated the influence of frequency band. GA, and level of fetal activity on the spectral integrals of FHR fluctuations. A significant $(p<0.00006)$ difference was obtained between the young and the mature fetuses examined. We conclude that it may be possible to probe the development of fetal ANS by means of spectral analysis of HRV. Visualizing the age difference (Figs. 6 and 7), it seems that the immature fetal ANS generates a large amount of unarrayed changes in HR, resulting in a power spectrum with about twice as much energy. compared with the mature and more stable ANS during the last period of gestation. both in sleep and active state.

Conclusion. The object of our research was to identify a parameter that may be associated with the development of the fetal ANS throughout pregnancy. The main result of the work presented here is that young $(\mathrm{GA}=23.5 \pm 1 \mathrm{wk})$ and mature pregnancies $(\mathrm{GA}=39.75 \pm 1.5 \mathrm{wk}$ ) present significantly different $(p<0.00006)$ power spectra of HRV. This observation might possibly be used to create a fetal ANS development test derived from the power spectra of HRV.

The young fetuses present a higher level of HRV spectral power compared with the mature fetuses, probably due to their less organized neural activity. It seems that an RP may be observed in the average integrals of HRV power spectrum only during sleep state of the fetuses, using short-term traces of heart rate. 
In the low-frequency range, the relation between the spectral density (D) and the frequency (f) obeys a power law similar to that for adults. It was determined to be $D \propto f^{-0.85}$ in both young and mature fetuses.

Applying fetal AECG recording followed by fetal ECG detection and spectral analysis of FHR fluctuation, it seems possible to determine the state of fetal activity and to obtain some information about the state of maturation of the ANS. However, the episodic nature of the fetal breathing, the wide range of activity states, and the immaturity of the ANS cause a large variability in the spectral power and in the width and location of the RP when apparent.

Based on previous studies, one does expect the high-frequency peak to be related to breathing activity; however, a choice of reliable criteria for the occurrence of an RP and its conjunction with breathing episodes requires an extensive specific investigation involving the simultaneous measurement of breathing movements by ultrasonic imaging, currently performed in our laboratory. The use of spectral analysis of FHR fluctuations as an investigative or clinical tool still requires extreme caution and extended research.

\section{REFERENCES}

1. Hyndman BW, Kitney RI. Sayers BM 1971 Spontaneous rhythms in physiological control systems. Nature 233:339-341

2. Pomeranz B, MaCauley RJB. Caudill MA. Kutz I, Adam D. Gordon D. Kilborn KM. Barger AC. Shannon DC. Cohen RJ. Benson H 1985 Assessment of autonomic function in humans by heart rate spectral analysis. Am J Physiol 248:H151-H153

3. Akselrod S. Gordon D. Madwed J. Snydman N. Shannon DC. Cohen RJ 1985 Hemodynamic regulation: investigation by spectral analysis. Am J Physiol 249:H867-H875

4. Lishner M, Akselrod S, Mor-Avi V, Oz O. Divon M, Ravid M 1986 Spectral analysis of heart rate fluctuations: a noninvasive, sensitive method for the early diagnosis of autonomic neuropathy in diabetes mellitus. J Auton Neur Syst 19:119-125

5. Akselrod S. Lishner M, Oz O, Bernheim J, Ravid M 1987 Spectral analysis of fluctuation in heart rate: an objective evaluation of autonomic nervous control in chronic renal failure. Nephron 45:202-206

6. Akselrod S, Arbel J, Oz O, Benary V. David D 1985 Evaluation of autonomous tone during acute myocardial infarction by spectral analysis of heart rate fluctuations. Circulation 72:242-246
7. Gordon D, Cohen RJ, Kelly D, Akselrod S, Shannon DC 1984 Sudden infant death syndrome: abnormalities in short-term heart rate and respiratory activity. Pediatr Res 18:921-926

8. Akselrod S, Gordon D, Ubel FA, Shannon DC, Berger RD, Cohen RJ 1981 Power spectrum analysis of heart rate fluctuations: a quantitative probe of beat-to-beat cardiovascular control. Science 213:220-2

9. Boddy K, Dawes GS 1975 Fetal breathing. Br Med B 31:3-7

10. Divon MY, Zimmer EZ, Platt LD, Paldi E 1985 Associated changes in hear rate and beat to beat variability. Am J Obstet Gynecol 151:403-406

11. Timor-Tritsch IE, Dierker LJ, Hertz RH, Chik L, Rosen MG 1980 Regular and irregular human fetal respiratory movement. Early Hum Dev 4:315324

12. Akselrod S, Karin J, Hirsch M 1987 Computerized detection of fetal ECG from maternal abdominal signal. IEEE (Inst Electr Electron Eng) Comp Cardiol 261-264

13. Shields JR, Schifrin BS 1988 Perinatal antecedents of cerebral palsy. Obste Gynecol 71:899-905

14. Castle BM, Tumbull AC 1983 The presence or absence of fetal breathing movements predicts the outcome of preterm labor. Lancet $2: 471-473$

15. Kobayashi M, Musha T 1982 1/f Fluctuations of heartbeat period. IEEE Trans Biomed Eng 29:480-481

16. Saul JP, Albrecht $P$, Berger RD, Cohen RJ 1987 Analysis of long term hear rate variability: methods, $1 / \mathrm{f}$ scaling and implications. IEEE (Inst Electr Electron Eng) Comp Cardiol 419-422

17. Bak P, Tang C, Wisenfeld K 1987 Self-organized criticality: an explanation of 1/f noise. Physical Rev Lett 59:381-384

18. Berger RD, Akselrod S, Gordon D, Cohen RJ 1986 An efficient algorithm for spectral analysis of heart rate variability. IEEE Trans Biomed Eng 33:900904

19. Oppenheim AV, Schaefer RW 1975 Digital Signal Processing. Prentice-Hall, Englewood Cliffs, NJ

20. Devoe LD, Ruedrich DA. Searle NS 1989 Value of observation of fetal breathing activity in antenatal assessment of high risk pregnancy. Am J Obstet Gynecol 160:166-171

21. Signorini MG, Agostoni O, Brina A, Cerutti S, Di Michele R, Pagani M 1990 Spectral analysis of heart rate variability signals in newborns: physiological and clinical aspects. IEEE (Inst Electr Electron Eng) Comp Cardiol 79-82

22. Aarimaa T, Oja R, Antila K, Valimaki I 1988 Interaction of heart rate and respiration in newborn babies. Pediatr Res 24:745-750

23. Baldzer K, Dykes FD, Jones SA, Brogan M. Carrigan TA, Giddens DP 1989 Heart rate variability analysis in full term infants: spectral index for study of neonatal cardiorespiratory control. Pediatr Res 26:188-195

24. Divon MY, Sze-Ya Y, Zimmer EZ, Platt LD, Paldi E, Paul RH 1985 Respiratory sinus arrhythmia in the human fetus. Am J Obstet Gynecol 151:425428

25. Dykes FD, Ahmann PA, Baldzer K, Carrigan A, Kitney RI, Giddens DP 1986 Breath amplitude modulation of heart rate variability in normal full term neonates. Pediatr Res 20:301-308

26. Kitney RI 1984 New findings in the analysis of heart rate variability in infants. Automedica 5:289-310

27. Giddens DP, Kitney RI 1985 Neonatal heart rate variability and its relation to respiration. J Theor Biol 113:759-780 